\title{
Current stage of studies of the star configurations at intermediate energies with the use of the BINA detector
}

A. Wilczek ${ }^{1 \star}$, A. Szadziński ${ }^{1}$, K. Bodek ${ }^{2}$, I. Ciepał ${ }^{3}$, N. Kalantar-Nayestanaki ${ }^{4}$, G. Khatri ${ }^{5}$, St. Kistryn ${ }^{2}$, B. Kłos ${ }^{1}$, A. Kozela ${ }^{3}$, J. Kuboś ${ }^{3}$, P. Kulessa ${ }^{6}$, A. Łobejko ${ }^{1}$, A. Magiera ${ }^{2}$, J. Messchendorp ${ }^{4}$, I. Mazumdar ${ }^{7}$, W. Parol $^{3}$, R. Ramazani-Sharifabadi ${ }^{4,8}$, D. Rozpȩdzik ${ }^{2}$, I. Skwira-Chalot ${ }^{9}$, E. Stephan ${ }^{1}$, B. Włoch ${ }^{3}$, A. Wrońska ${ }^{2}$, and J. Zejma ${ }^{2}$

1 Institute of Physics, University of Silesia, Chorzow, Poland

2 Institute of Physics, Jagiellonian University, Krakow, Poland

3 Institute of Nuclear Physics, PAS, Krakow, Poland

4 KVI-CART, University of Groningen, Groningen, The Netherlands

5 Department of Physics and Astronomy, Northwestern University, Evanston, USA

6 Forschungszentrum Jülich, Institut für Kernphysik, Jülich, Germany

7 Tata Institute of Fundamental Research, Mumbai, India

8 Department of Physics, University of Tehran, Tehran, Iran

9 Faculty of Physics University of Warsaw, Warsaw, Poland

* awilczek@us.edu.pl

October 26, 2019

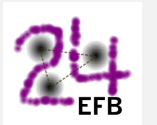

Proceedings for the 24th edition of European Few Body Conference, Surrey, UK, 2-4 September 2019

\begin{abstract}
The Space Star Anomaly in proton-deuteron breakup cross-section occurs at energies of about $10 \mathrm{MeV}$. Data for higher energies are sparse. Therefore, a systematic scan over star configurations in the range of intermediate energies between 50 and $100 \mathrm{MeV} /$ nucleon is carried out on the basis of data collected with the large acceptance BINA detector. The preliminary cross section results for forward star configurations at $80 \mathrm{MeV} /$ nucleon slightly surpass the theoretical calculations, but the systematic uncertainties are still under study. Also, a new variable describing rotation of star configurations is proposed.
\end{abstract}

\section{Introduction}

Studies of the most basic properties of nuclear interactions require a system simple enough for the strict calculations to be performed, which at the same time should be complicated enough to make some additional dynamics possible. These conditions are fulfilled by fewbody systems. The system of three nucleons $(3 \mathrm{~N})$ is the simplest non-trivial environment, in which the nucleon-nucleon (NN) interactions models can be tested. Although NN interactions are dominant in the few-nucleon interactions, the system composed of at least 3 nucleons can not be properly described without introducing additional dynamic ingredient - three-nucleon force (3NF).

State-of-the-art theoretical descriptions of nuclear interactions usually use either of two theoretical approaches in modelling the dynamics: realistic potentials or Effective Field 
Theory (EFT), as it is not possible to directly apply QCD in this energy region. Phenomenological NN potentials are either complemented with additional diagrams treating excitations on an equal footing with nucleons, as if stable particles were produced $(\Delta$ isobar in Coupled Channels approach) or with a phenomenological 3NF model. Another approach is more fundamental and applies the Chiral Perturbation Theory in order to reach an EFT description (Chiral EFT) [1,2]. Recent developments of the calculations with realistic potentials involve relativistic effects [3,4] as well as Coulomb interactions [5].

Even though the development of the theories is well advanced, some experimentally discovered effects are still awaiting their correct theoretical description. The discrepancies between the theory and the experiment were observed both in cross sections and in polarisation-dependent observables. The most intriguing inconsistencies are the $A_{y}$ puzzle (for analysing powers) and the Space Star Anomaly (SSA) observed in the differential cross-section of the breakup reaction. The latter effect is surprising, as it occurs at relatively low energies. The effect has not been explained theoretically since its discovery in 1989 [6]. The star configurations are defined in the centre of mass system as a final state of the $d+p \rightarrow p+p+n$ reaction, where the momenta of the outgoing nucleons form an equilateral triangle. Depending on the angle of inclination with respect to the beam axis $(\alpha)$, one defines the Space Star $\left(\alpha=90^{\circ}\right)$, Forward Plane Star (with neutron momentum pointing upstream the beam, $\alpha=0^{\circ}$ ), and Backward Plane Star (with neutron moving in the beam direction, $\alpha=180^{\circ}$ ). The biggest discrepancies for the Space Star configuration are observed at energies ranging from 7.5 to $13 \mathrm{MeV} /$ nucleon, where the differences reach $15 \%$ [7], and the theories overestimate the measurements. The main component of the theoretical cross-sections at such low energies results from the s-wave channel of the NN interaction, what reduces the range of possible explanations for the effect. Expected 3NF and Coulomb effects are very small and they were applied to theories, without clarifying the observed inconsistency. At so low energy, relativistic effects are negligible. Up till now, the calculations do not reproduce the cross-sections for the Space Star Anomaly in reliable way. Interestingly, the discrepancy is twice as much in neutron-deuteron breakup, where the experimental cross-sections exceed by $30 \%$ the theoretical predictions $[8,9]$. In other words, the effect switches the sign under isospin change. A set of very precise cross-section measurements for breakup, in both $p+d$ and $n+d$ collisions in a wide range of energy is needed to solve this charge-symmetry breaking puzzle.

The set of differential cross-sections for the ${ }^{1} H(d, p p) n$ and ${ }^{2} H(p, p p) n$ reactions collected with the large acceptance BINA detector is used for scanning the star configurations over a wide range of beam energies between 50 and $160 \mathrm{MeV} /$ nucleon. The main aim is to check if the effect is still measurable for energies within this range. The only published data about the Space Star for this energy range showed lack of the effect for $65 \mathrm{MeV}$ [10], while for the second highest energy $(19 \mathrm{MeV})$ the effect was small, but still visible [11].

\section{The BINA experimental setup}

Currently, the Big Instrument for Nuclear Polarization Analysis (BINA) detector setup is placed in Cyclotron Centre Bronowice (CCB), PAS Krakow [12]. A liquid hydrogen or deuterium target is placed in the centre of a scattering chamber made of 149 phoswich detectors of triangular cross-section, called Ball. Ball covers polar angles between $40^{\circ}$ and $160^{\circ}$ with angular resolution of about $10^{\circ}$. The second part of the detector is called Wall. It consists of Multi-Wire Proportional Chamber (MWPC), thin rectangular scintillators for energy loss measurement $(\Delta \mathrm{E})$ and a calorimeter made of thick scintillator blocks (E). Combination of signals from $\mathrm{E}$ and $\Delta \mathrm{E}$ is used for particle identification. Track 
reconstruction for forward angles is based upon the MWPC employing 3 detection layers. The Wall covers polar angles $\theta \in\left(10^{\circ}, 35^{\circ}\right)$ and its angular resolution is of about $0.5^{\circ}$. Such a combination of detectors covers a solid angle of almost $4 \pi$, what makes the system well suited to the measurements of variety of star geometries.

The experiments performed with BINA at KVI, Groningen, and the ongoing measurements at CCB, Krakow, make possible to extend the experimental data on the Space Star cross section at 50, 80, 108 and $160 \mathrm{MeV} /$ nucleon. New measurements (proton beam at 108, 135 and $160 \mathrm{MeV}$ ) were performed in August/October 2019 and will be analysed soon.

\section{Results}

The preliminary results (Fig. 1) at 3 different inclination angles $\alpha$ are obtained with the data taken at KVI Groningen in 2011 for $\mathrm{d}+\mathrm{p} \rightarrow \mathrm{p}+\mathrm{p}+\mathrm{n}$ reaction at $80 \mathrm{MeV} /$ nucleon. The angle $\alpha=20^{\circ}$ corresponds to polar angles (in laboratory system) of registered protons $\theta_{1}=\theta_{2}=24.2^{\circ} \pm 1^{\circ}$ and relative azimuthal angle $\phi_{12}=157.7^{\circ} \pm 5^{\circ} ; \alpha=40^{\circ}$ corresponds to $\theta_{1}=\theta_{2}=26.3^{\circ} \pm 1^{\circ}$ and $\phi_{12}=139.3^{\circ} \pm 5^{\circ} ; \alpha=60^{\circ}$ corresponds to $\theta_{1}=\theta_{2}=29.2^{\circ} \pm 1^{\circ}$ and $\phi_{12}=126.9^{\circ} \pm 5^{\circ}$. The analysis $[13,14]$ relies on reconstruction of proton energies, polar angles and a relative azimuthal angle of the proton pair. It includes correction for detector efficiency, cross-over regions, as well as normalisation to the luminosity. For the star configurations, the correction for loss of coincidences due to double-hits in the same elements has not been implemented yet. The missing efficiency factor depends on angular configuration only, thus, it is energy independent. Preliminary estimations provide the correction factor of about $5 \%$, so the presented cross-section distributions should be increased by roughly $0-10 \%$. Simulations of the losses due to double-hits in all the detector elements and analysis of the systematic uncertainties are under way. 3NF effects are small and Coulomb effects are negligible (see Fig. 1). Relativistic effects should be included, as they account for measurable effect even at $65 \mathrm{MeV}$ [10]

The symmetry axis in Fig. 1, corresponds to star configurations. The other points are measured for the same angular configuration of protons, but with asymmetric momentum distribution between them. All the presented data show coincidences of two particles registered in the Wall. This makes possible to check the feasibility of providing new good-quality results on star configurations. The cross-section for the star configurations in deuteron on proton breakup increases with $\alpha$, until it reaches the value corresponding to $\alpha=180^{\circ}$, which is about 3 times higher than for $\alpha=20^{\circ}$, what makes possible to reduce statistical uncertainties. On the other hand, $\alpha>140^{\circ}$ correspond to Ball-Ball coincidences, which due to worse angular resolution are subject to higher systematic error.

\section{New variable - rotation in the plane}

Standard analysis of star configurations with respect to $\alpha$, in case of proton beam and the BINA detector leads to the following picture (Fig. 3, region near $\beta=0^{\circ}$ ):

- quite few star configurations are covered by Wall-Wall coincidences (the highest angular resolution),

- Ball-Wall coincidences (intermediate angular resolution) have no contributions,

- the angles between $\alpha=50^{\circ}$ up to $\alpha=180^{\circ}$ can be registered as Ball-Ball coincidences (the lowest angular resolution). 


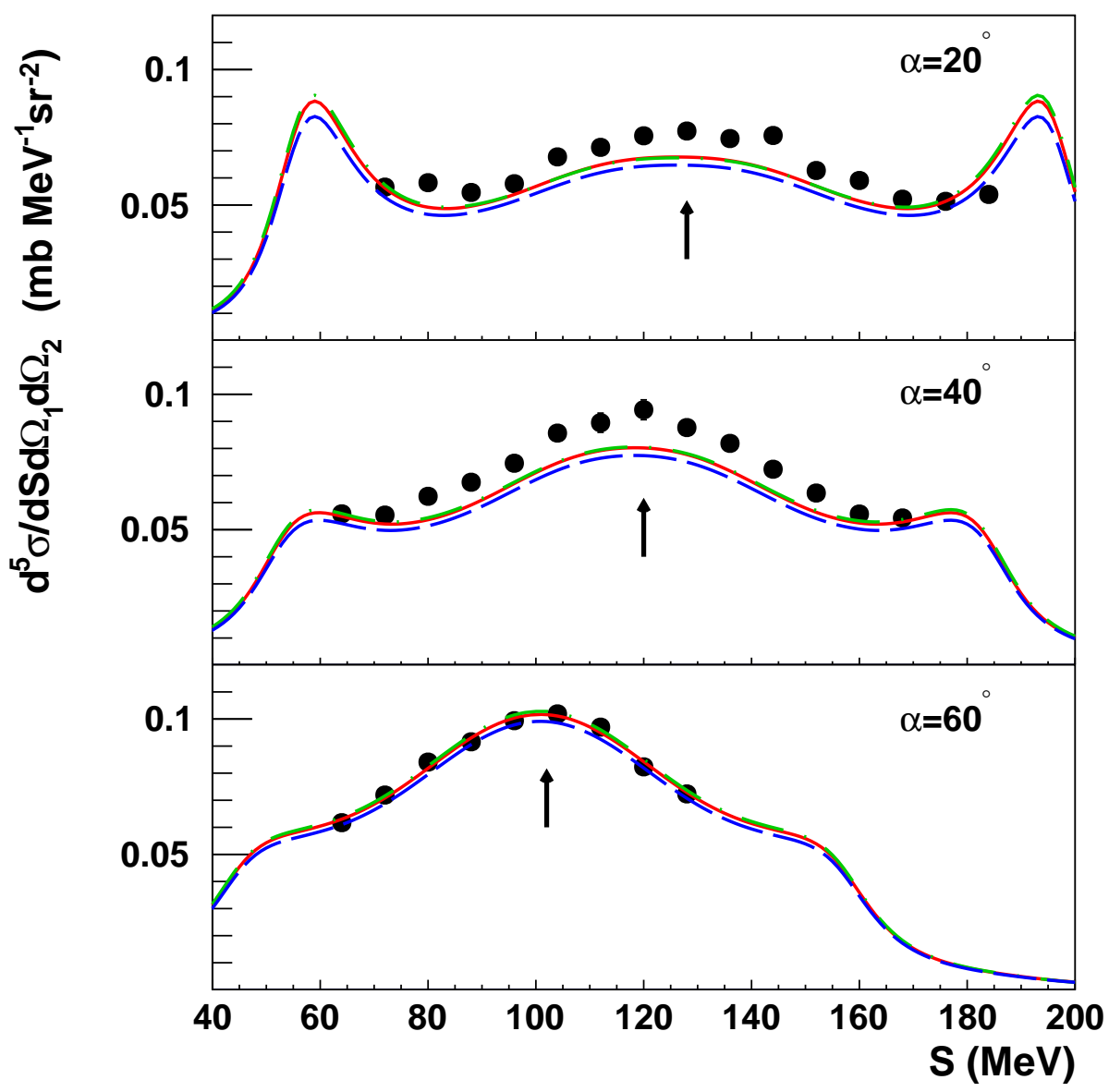

Figure 1: Deuteron breakup cross-section $(\mathrm{d}(160 \mathrm{MeV})+\mathrm{p} \rightarrow \mathrm{p}+\mathrm{p}+\mathrm{n})$ for 3 different values of $\alpha$. Cross-section distributions are plotted against the value of the arc length along the kinematics $(S)$ with the starting point $(S=0)$ chosen arbitrarily at the point where $E_{2}$ reaches minimum (see Fig. 2). The central value corresponds to the star configuration (arrow). The theoretical predictions are plotted as lines (red solid: CD-Bonn $+\Delta+$ Coulomb, green chain: CD-Bonn $+\Delta$, blue dashed: CD-Bonn+Coulomb) $[5,15]$. The normalisation of the experimental data is not finalised and does not contain e.g. effects due to with double-hits. 


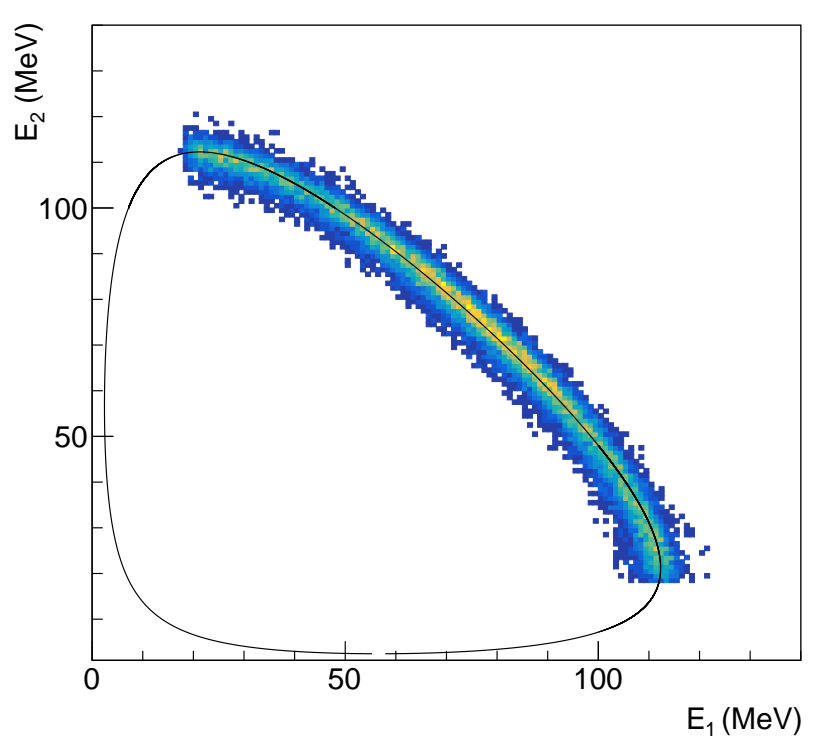

Figure 2: An example of kinematics for $(\mathrm{d}(160 \mathrm{MeV})+\mathrm{p} \rightarrow \mathrm{p}+\mathrm{p}+\mathrm{n})$ reaction at $\alpha=20^{\circ}$. Selection of polar and azimuthal angles implies strict kinematic relation between kinetic energies of emitted protons.

The way for improvement is paved by an application of another variable (Fig. 3). In addition to $\alpha$ one can define $\beta$ angle, which is the measure of rotation about the axis perpendicular to the plane spanned by the momenta of the reaction products. The axis of the rotation originates at the reaction vertex. The rotation through $\beta=120^{\circ}$ results in a configuration, where neutron and proton are exchanged - e.g. in the case of Forward Plane Star $\left(\alpha=0^{\circ}\right)$ neutron will be registered at the same angle, as one of the protons, whereas the second proton will move in the direction against the beam in the centre of mass system.

Applying rotation through $\beta$ angle one can measure Ball-Wall coincidences in the region not accessible to the standard approach. Although it is still not possible to improve resolution for detecting the Space Star configuration $\left(\alpha=90^{\circ}\right)$, the regions between $\alpha=0^{\circ}$ and $\alpha=60^{\circ}$, as well as between $\alpha=130^{\circ}$ and $\alpha=180^{\circ}$ are within the acceptance of the BallWall coincidences.

\section{Conclusion}

Although the BINA energy scan is very promising, the current state of analysis does not allow to draw a clear conclusion about the Space Star Anomaly, as the configuration at $\alpha=90^{\circ}$ has not been analysed yet. In order to reach the expected number of configurations altogether with the Space Star one has to develop analysis for Ball-Ball coincidences. The analysis of Wall-Wall coincidences is about to be finalised. Preliminary data show required statistical accuracy and their shape is correctly reproduced by stat-of-the-art calculations. The normalisation is to be corrected for loss of data due to double-hits and the systematic effects have to be studied in detail. Scope of the studies can be extended by applying a newly proposed variable, $\beta$, which enables to analyse numerous configurations at the same $\alpha$ angle simultaneously. In addition to that, new measurements of the breakup 

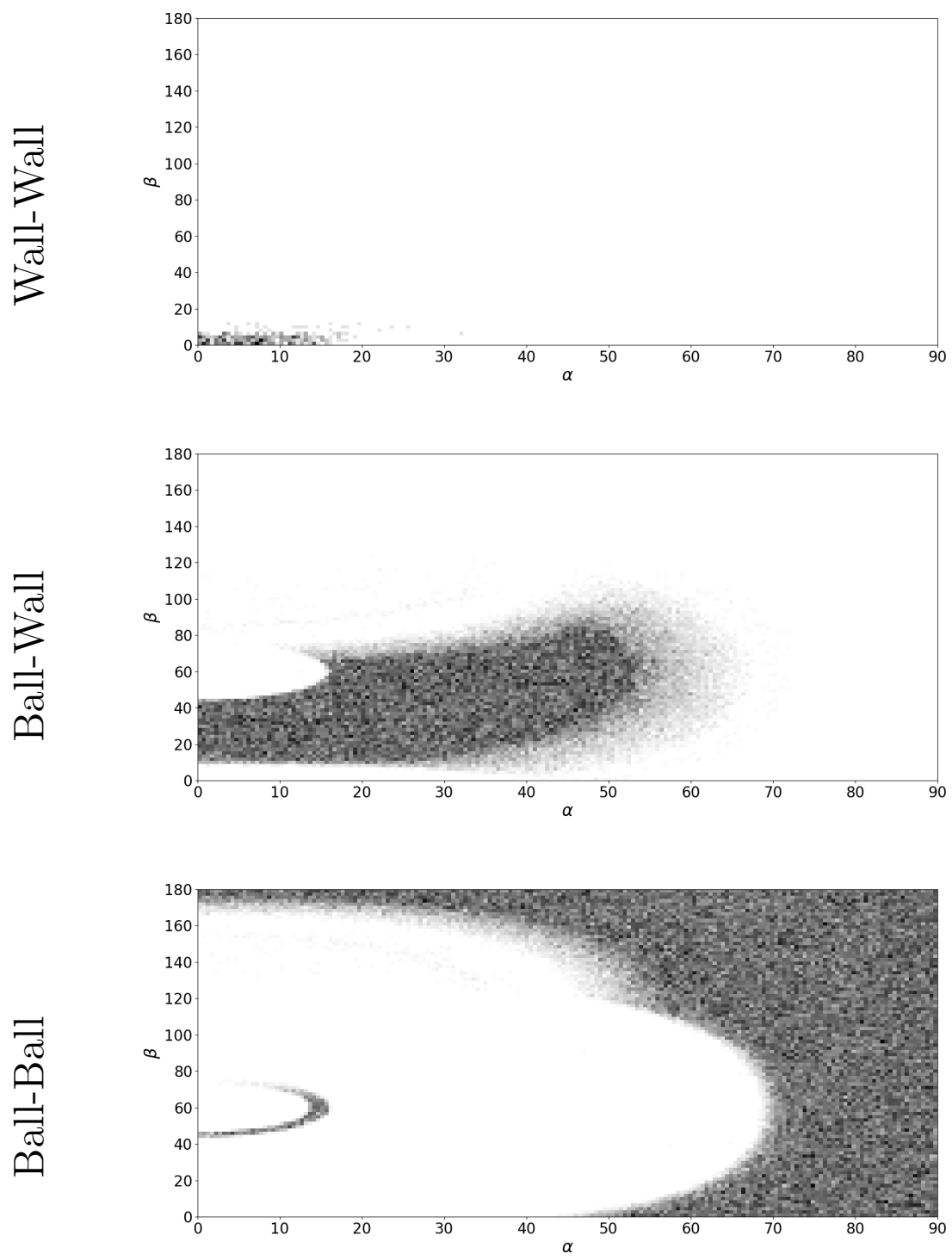

Figure 3: Simulated acceptance of the BINA detector for $\mathrm{p}(160 \mathrm{MeV})+\mathrm{d} \rightarrow \mathrm{p}+\mathrm{p}+\mathrm{n}$. 3 different kinds of coincidence: Wall-Wall $($ top $)$, Ball-Wall (middle), Ball-Ball (bottom). The axes are scaled in degrees. 
reaction with 108 and $160 \mathrm{MeV}$ proton beam will be conducted in near future.

\section{Acknowledgements}

Funding information This project is supported by the National Science Centre, Poland (grants nr. 2016/23/D/ST2/01703, and 2012/05/B/ST2/02556).

\section{References}

[1] E. Epelbaum, A. Nogga et al., Three-nucleon forces from chiral effective field theory, Phys. Rev. C 66, 064001 (2002), doi:10.1103/PhysRevC.66.064001

[2] E. Epelbaum, W. Glöckle, U.-G. Meissner, Improving the convergence of the chiral expansion for nuclear forces - I: Peripheral phases, Eur. Phys. J. A 19, 125 (2004) doi:10.1140/epja/i2003-10096-0

[3] H. Witala, J. Golak, R. Skibinski, Selectivity of the nucleon-induced deuteron breakup and relativistic effects, Phys. Lett. B 634, 374 (2006), doi:10.1016/j.physletb.2006.01.070.

[4] H. Witala et al., Three-nucleon force in relativistic three-nucleon Faddeev calculations, Phys. Rev. C 83, 044001 (2011), doi:10.1103/PhysRevC.83.044001.

[5] A. Deltuva, Momentum-space calculation of proton-deuteron scattering including Coulomb and irreducible three-nucleon force, Phys. Rev. C 80, 064002 (2009), doi:10.1103/PhysRevC.80.064002.

[6] J. Strate et al., Differential cross section of the ${ }^{2} H(n, n n p)$-reaction at $E_{n}=13 \mathrm{MeV}$, Nucl.Phys. A 501, 51 (1989), doi:10.1016/0375-9474(89)90564-2.

[7] K. Ohnaka et al., Systematic Measurement of pd Breakup Cross Section Around Space Star, Few-Body Syst. 55, 725 (2014), doi:10.1007/s00601-014-0890-7.

[8] K. Sagara, Recent Few-Nucleon Experiments and Discrepancies to be Solved, FewBody Syst. 55, 1073 (2014), doi:10.1007/s00601-014-0888-1.

[9] Z. Zhou, The space-star anomaly in nd breakup at $25 \mathrm{MeV}$, Nucl. Phys. A 684, 545 (2001), doi:10.1016/S0375-9474(01)00389-X.

[10] J. Zejma et al., Cross sections and analyzing powers $A_{y}$ in the breakup reaction ${ }^{2} H(\vec{p}, p p) n$ at 65 MeV: Star configurations, Phys. Rev. C 55, 42 (1997), doi:10.1103/PhysRevC.55.42.

[11] J. Ley et al., Cross sections and tensor analyzing powers $A_{y y}$ of the reaction ${ }^{1} \mathrm{H}(\vec{d}$, pp) $n$ in "symmetric constant relative energy" geometries at $E_{d}=19 \mathrm{MeV}$, Phys. Rev. C 73, 064001 (2006), doi:10.1103/PhysRevC.73.064001.

[12] St. Kistryn, E. Stephan, Deuteron-proton breakup at medium energies, J. Phys. G 40, 063101 (2013), doi:10.1088/0954-3899/40/6/063101. 
[13] W. Parol, Investigation of three-nucleon force effects in the reaction of deuteron proton breakup at the energy of $80 \mathrm{MeV} /$ nucleon (in Polish), Ph.D Thesis, Jagiellonian University, Krakow (2015) https://fais.uj.edu.pl/documents/41628/ 761d2b13-faa4-4960-bfe9-96157aa48bd6.

[14] W. Parol et al. Investigation of Three Nucleon Force Effects in Deuteron-Proton Breakup Reaction, Acta Phys. Pol. B 45, 527 (2014) doi:10.5506/APhysPolB.45.527

[15] A. Deltuva, A. C. Fonseca, P. U. Sauer, Momentum-space description of three-nucleon breakup reactions including the Coulomb interaction, Phys. Rev. C 72, 054004 (2005), doi:10.1103/PhysRevC.72.054004 